\title{
Ecological changes in the Southern California Bight near a large sewage outfall: benthic conditions in 1980 and 1983
}

\author{
R. C. Swartz, F. A. Cole, D. W. Schults \& W. A. DeBen \\ U.S. Environmental Protection Agency, Mark O. Hatfield Marine Science Center, Newport, Oregon 97365, USA
}

\begin{abstract}
The structure of the macrobenthic community, sediment toxicity, and sediment contamination changed greatly between 1980 and 1983 along a pollution gradient from the Los Angeles County Sanitation Districts' (LACSD) sewage outfalls on the Palos Verdes Shelf, California, USA to a reference site in northern Santa Monica Bay. Stimulation of the species richness, biomass, and density of the benthos along the $60 \mathrm{~m}$ contour 5 to $11 \mathrm{~km}$ from the outfalls was significantly reduced in 1983 when compared to 1980 . The clam Parvilucina tenuisculpta and the polychaete Tharyx sp. A were the numerically dominant species in the area of benthic stimulation in both years. Stations 1 to $3 \mathrm{~km}$ from the outfalls in 1980 were occupied by a depauperate fauna strongly dominated by the opportunistic polychaete Capitella spp. In 1983 species richness and biomass significantly increased close to the outfalls, Capitella spp. was much less abundant, and the assemblage was dominated by ostracods of the genus Euphilomedes that are characteristic of background conditions or areas only slightly affected by wastewater discharges. At the reference site in Santa Monica Bay there were no significant changes in species richness, biomass, or density between 1980 and 1983, and the brittlestar Amphiodia urtica remained the dominant species. Sediment contamination by most measured chemicals and parameters of organic enrichment decreased on the Palos Verdes Shelf, but not at the reference station, between 1980 and 1983. Sediments collected within $3 \mathrm{~km}$ of the outfalls in 1980 were acutely toxic to the phoxocephalid amphipod Rhepoxynius abronius. There was no significant toxicity associated with any sediment from the Shelf in 1983. These ecological changes correspond with the reduction in the mass emission of BOD and chemical contaminants from the LACSD outfalls between 1980 and 1983. Severe storms in the winter of $1982-83$ may also have contributed to improvements in sediment quality and the partial recovery of the macrobenthos.
\end{abstract}

\section{INTRODUCTION}

The marine macrobenthos responds in a consistent pattern to changes in the level of sediment organic enrichment (Pearson \& Rosenberg 1978). Benthic assemblages near major sources of organic material contain no macrofauna or only a few pollution-tolerant, opportunistic species that may be very abundant. At sites further from the enrichment source, the opportunists are replaced by an assemblage of greater species richness and biomass that gradually changes into the community characteristic of the unpolluted environment. The Pearson \& Rosenberg (1978) model applies to successional patterns that occur in both time and space in response to increasing or decreasing sediment organic enrichment. The model is consistent with benthic spatial distributions near sewage outfalls and the temporal response of the benthos to the initiation or elimination of sewage discharges in open coastal waters (Smith 1974, Anger 1975, Smith \& Greene 1976. Bascom 1978, 1982, LACSD 1981, Mearns \& Word 1982, Swartz et al. 1985a, Stull et al. 1986). However, little is known about benthic responses to incremental changes in the mass emission of organic material and specific chemicals. We have, therefore, examined sediment contamination, sediment toxicity, and benthic community structure near a major sewage outfall during a period of gradual reduction in the mass emission of many effluent constituents.

The Los Angeles County Sanitation Districts (LACSD) discharge approximately $5 \times 10^{11} \mathrm{l} \mathrm{yr}^{-1}$ of municipal wastes on the Palos Verdes Shelf, California, USA (Schafer 1984). Sufficient particulate material accumulates on the seabed to greatly increase the sediment concentration of chemical contaminants and organic matter. These sediment changes have substan- 
tially altered the macrobenthos in a pattern predicted by the Pearson \& Rosenberg (1978) model (Swartz et al. 1985a). Since at least 1971, benthic species richness, biomass, and density over a large area of the Shelf have been significantly greater than background conditions (SCCWRP 1973). At sites closer to the outfalls there have been significant reductions in these parameters and a strong numerical dominance by the opportunistic polychaete, Capitella spp.

Since 1971 there has been a gradual decline in the mass emission of suspended solids and most chemical contaminants from the LACSD outfalls (LACSD 1981, Stull et al. 1986). Between 1977 and 1980 the average reduction in the emission of the 22 effluent components monitored by Schafer $(1978,1980,1984)$ was $14.2 \%$ and from 1980 to 1983 the average reduction was $18.6 \%$.

In 1983, we repeated the survey conducted in 1980 (Swartz et al. 1985a) of the macrobenthos, sediment toxicity and chemical contamination on the Palos Verdes Shelf. Our objective was to determine if the reduction in mass emissions from the outfalls resulted in a reduction in sediment contamination and a recovery of the macrobenthos. We examined 5 a priori predictions, related to the Pearson \& Rosenberg (1978) model, of ecological changes that would occur in response to improved sediment quality: (1) the abundance, richness, and biomass of the benthos at the site of maximum stimulation in 1980 (Stn 6) would be reduced; (2) the site of maximum benthic stimulation would move closer to the outfalls; (3) tolerant, opportunistic species would disappear or become less abundant at sites closest to the outfalls; (4) less tolerant species would appear or be more abundant at sites closer to the outfalls; and (5) the slight sediment toxicity detected in 1980 near the outfalls would be reduced or eliminated. The relative tolerance of benthic species to sediment organic enrichment was based on Word's $(1978,1980)$ classification of 45 taxa according to their distribution near sewage outfalls in the Southern California Bight.

\section{MATERIALS AND METHODS}

Sediment and macrobenthos samples were collected on the Palos Verdes Shelf and Santa Monica Bay, California on 13 to 15 May 1980 at Stns $1,2,3,4,6,8$ and 9 , and on 27 to 29 June 1983 at Stns $1,3,4,5,6,7,8$ and 9 (Fig. 1). Stn 2 was not occupied in 1983 because benthic conditions there were very similar to those at Stn 1 in 1980 (Swartz et al. 1985a). Stns 5 and 7 were added in 1983 to provide better spatial coverage of ecological changes on the Shelf. The LACSD outfalls discharge at a water depth of approximately $60 \mathrm{~m}$ on the southeast portion of the Shelf. The first 8 stations

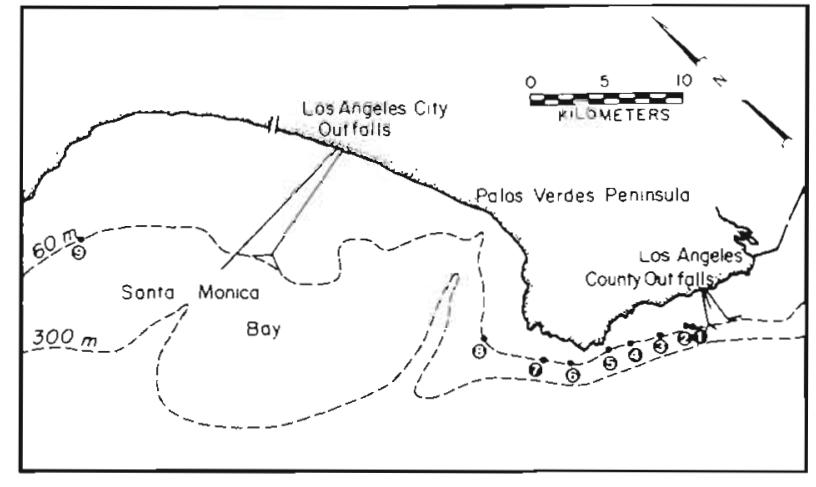

Fig. 1. Location of benthic stations on the Palos Verdes Shelf and Santa Monica Bay, California

were located along the $60 \mathrm{~m}$ contour at increasing distances of 1 to $15 \mathrm{~km}$ to the northwest of the outfalls in the direction of predominant benthic deposition of sewage particles (LACSD 1981, Hershelman et al. 1981). Stn 9 was $47 \mathrm{~km}$ from the outfalls at a water depth of $60 \mathrm{~m}$ in northern Santa Monica Bay. This reference station is the closest site to the study area that is representative of background benthos conditions in the southern California Bight (Word \& Mearns 1978, Bascom 1978). The Los Angeles City sewage outfalls in Santa Monica Bay have little or no influence on the pollution gradient ( 1 to 8 ) or reference (9) stations (Bascom et al. 1978).

Ten $0.1 \mathrm{~m}^{2}$ van Veen grab samples were collected at each station. The contents of 5 of the grabs were sieved through a $1.0 \mathrm{~mm}$ mesh screen to collect macrofauna. Material retained on the screen was preserved in buffered $10 \%$ formalin, later transferred to $70 \%$ ethanol, sorted to major phylogenetic group, and weighed $( \pm 0.1 \mathrm{~g})$ after blotting on absorbent paper. Specimens were counted and usually identified to the species level. Polychaetes identified by taxonomic keys as Capitella capitata are referred to as Capitella spp. because of the possible occurrence of sibling species in our collections (e.g. Grassle \& Grassle 1976). Certain alphabetic codes for distinct species reflect assignments given by the Southern California Association of Marine Invertebrate Taxonomists, e.g. Tharyx sp. A. and Myriochele sp. M.

The structure of the benthic community was analyzed in relation to species composition, species richness (number of species, $\mathrm{S}$, collected per $0.1 \mathrm{~m}^{2}$ grab), density (number of individuals, $\mathrm{N}$, per $0.1 \mathrm{~m}^{2}$ ), biomass (g per $0.1 \mathrm{~m}^{2}$, wet weight), dominance, and Infaunal Index. The dominance index was defined as the minimum number (or fraction) of species whose combined abundance was equal to $75 \%$ of the individuals in the sample. The dominance index is inversely related to numerical dominance. The Infaunal Index is based on the relative abundance of 4 groups of species 
representing different stages of macrobenthic succession in relation to organic enrichment near sewage outfalls in the southern California Bight. Group I includes species that are dominant in background areas and decrease in abundance with increasing proximity to sewage outfalls (e.g. phoxocephalid and ampeliscid amphipods, ophiuroids). Species of Group II increase in abundance in areas slightly affected by sewage discharges (e.g. Euphilomedes spp., Mediomastus spp.). Species of Group III are present in moderately affected areas (e.g. Parvilucina tenuisculpta, Macoma carlottensis). Opportunistic species found in heavily affected areas are placed in Group IV (e.g. Capitella spp., Schistomeringos spp.). The complete list of species in each of the 4 groups and the index formula are given by Word $(1978,1980)$ and Mearns \& Word (1982). Index values range from 0 when all individuals belong to opportunistic species of Group IV to 100 when all individuals belong to Group I.

Samples from the remaining 5 grabs were used for physical, chemical, and toxicological analyses. Methods are described in detail by Swartz et al. (1985a). The van Veen grab was equipped with hinged top plates that permitted sampling of the sediment surface. Redox potential (Eh) was measured by inserting a $\mathrm{Pt} /$ calomel electrode $1 \mathrm{~cm}$ beneath the sediment surface. Interstitial water was collected by suction into a syringe through a cellulose filter inserted into the sediment, and preserved for ammonia-nitrogen and free sulfide analyses. Cores $(2 \mathrm{~cm}$ deep) were taken from the surficial sediment layer in each grab for analysis of (1) sediment toxicity, (2) total volatile solids and total organic carbon, (3) $5 \mathrm{~d}$ sediment BOD, (4) total oil and grease, hydrocarbon oil and grease, (5) total sulfide, (6) metals $(\mathrm{Cd}, \mathrm{Pb}, \mathrm{Zn}, \mathrm{Cr}, \mathrm{Cu}, \mathrm{Ni}),(7)$ bis(2-ethylhexyl) phthalate (DEHP), and (8) 4,4'-DDE. DDE and DEHP were measured in only 2 grabs from each station during the 1980 survey. All other parameters were measured in 5 replicate grab samples from each station in 1980 and 1983 . The bulk concentration of all chemicals in sediment is expressed on a dry weight basis. Sediment toxicity was determined by the survival of the phoxocephalid amphipod Rhepoxynius abronius after $10 \mathrm{~d}$ of exposure to Palos Verdes sediment (Swartz et al. 1985b). Sediment from the Yaquina Bay, Oregon, USA collection site of $R$, abronius was used as a reference for the sediment bioassays.

Statistical comparisons of differences in mean values between stations and between years for biological and chemical parameters were made by t-tests, analysis of variance, Student-Newman-Keuls multiple range test, and, when variance was heterogenous for transformed data, the Games and Howell method (Sokal \& Rohlf 1981, Steel \& Torrie 1960). $F_{\max }$ was used to test for homogeneity of variance. Log transformations were applied when variances were heterogenous. Dunnett's procedure was used to compare mean survival in sediment from the Palos Verdes Shelf with mean survival in the Yaquina Bay reference sediment (Steel \& Torrie 1960). Unless otherwise noted, all statements about statistical comparisons are based on the $p<0.05$ significance level.

The biological and chemical data sets were analyzed separately by numerical classification techniques to establish the relative similarity of individual grab samples without consideration of the station or year of collection. Samples were clustered on the basis of similarity in the composition and abundances of macrobenthic species or the presence and magnitude of chemical parameters. The Canberra metric dissimilarity coefficient and the flexible sorting strategy of Lance \& Williams (1966) with $\beta=-0.25$ were used in the classification analyses (Boesch 1977). To reduce the biological data set to a manageable size, rare species represented by a total of less than 20 individuals in all samples were excluded from the classification. All measured chemical parameters except DDE and DEHP were included in the chemical classification. DDE and DEHP were excluded because they were analyzed in only 2 samples from each station in 1980.

\section{RESULTS}

\section{Dominant macrobenthic species: 1980-1983 comparison}

The composition and abundance of dominant species changed between 1980 and 1983 at stations close to the outfalls (Table 1). The 5 most abundant species at Stn 1 in 1980 were the polychaetes Capitella spp., Nereis procera, Nephtys cornuta franciscana, Eumida sanguinea, and Schistomeringos longicornis. All of these species disappeared or declined in abundance at $\operatorname{Stn} 1$ in 1983. The proportion of individuals belonging to Capitella spp. decreased at Stn 1 from $85.2 \%$ in 1980 to $10.8 \%$ in 1983 . The dominants at Stn 1 in 1983 included the ostracod Euphilomedes spp., the polychaetes Tharyx sp. A, Capitella spp., and Mediomastus californiensis, and the pelecypod Tellina modesta. In 1980, all of these species except Capitella spp. had densities $<10.1 \mathrm{~m}^{-2}$ at Stn 1 and were more abundant at stations further from the outfalls. Temporal changes at $\operatorname{Stn} 3$ were similar to those at $\operatorname{Stn} 1$. The proportion of individuals belonging to Capitella spp. declined from $56.2 \%$ in 1980 to $0.1 \%$ in 1983 . With the exception of the pelecypod Parvilucina tenuisculpta, the 5 most abundant species in 1980 were less abundant in 1983, and the 1983 dominants were 
rare in 1980 . In 1983, 4 of the 5 most abundant dominants were the same species at both Stns 1 and 3 .

The composition of the dominant species at stations $5 \mathrm{~km}$ or more from the outfalls did not change greatly between 1980 and 1983. Tharyx sp. A, Parvilucina tenuisculpta and Mediomastus californiensis were consistently among the 5 most abundant species at Stns 4, 6, and 8 in 1980 and 1983 and at Stns 5 and 7 that were sampled only in 1983 (Table 1). Although they remained strong dominants, these 3 species were

Table 1. Mean densities of the 5 most abundant species at at least 1 station on the Palos Verdes Shelf and Santa Monica Bay, California in 1980 or 1983 . Five replicate $0.1 \mathrm{~m}^{2}$ grabs were taken at each station

\begin{tabular}{|c|c|c|c|c|c|c|c|c|c|c|}
\hline \multirow[t]{2}{*}{ Species } & \multirow[t]{2}{*}{ Year } & \multicolumn{9}{|c|}{ Station } \\
\hline & & 1 & 2 & 3 & 4 & 5 & 6 & 7 & 8 & 9 \\
\hline Eumida sanguinea (PO) & $\begin{array}{l}1980 \\
1983\end{array}$ & $\begin{array}{l}7.4 \\
0\end{array}$ & $\begin{array}{l}15.2 \\
\text { nd }\end{array}$ & $\begin{array}{l}0.6 \\
0.2\end{array}$ & $\begin{array}{l}0 \\
0\end{array}$ & $\begin{array}{l}\text { nd } \\
0.4\end{array}$ & $\begin{array}{l}0 \\
0.8\end{array}$ & $\begin{array}{l}\text { nd } \\
1.6\end{array}$ & $\begin{array}{l}0 \\
0.4\end{array}$ & $\begin{array}{l}0 \\
0\end{array}$ \\
\hline Schistomeringos longicornis (PO) & $\begin{array}{l}1980 \\
1983\end{array}$ & $\begin{array}{l}6.6 \\
0\end{array}$ & $\begin{array}{l}8.6 \\
\text { nd }\end{array}$ & $\begin{array}{l}1.4 \\
0\end{array}$ & $\begin{array}{l}0 \\
0\end{array}$ & $\begin{array}{c}\text { nd } \\
0\end{array}$ & $\begin{array}{l}0 \\
0\end{array}$ & $\begin{array}{c}\mathrm{nd} \\
0\end{array}$ & $\begin{array}{l}0 \\
0\end{array}$ & $\begin{array}{l}0 \\
0\end{array}$ \\
\hline Nephtys cornuta franciscana (PO) & $\begin{array}{l}1980 \\
1983\end{array}$ & $\begin{array}{l}7.8 \\
1.6\end{array}$ & $\begin{array}{l}3.8 \\
\text { nd }\end{array}$ & $\begin{array}{l}0.8 \\
1.2\end{array}$ & $\begin{array}{l}0.2 \\
0.2\end{array}$ & $\begin{array}{l}\text { nd } \\
0.8\end{array}$ & $\begin{array}{l}0.2 \\
0.4\end{array}$ & $\begin{array}{l}\mathrm{nd} \\
0.2\end{array}$ & $\begin{array}{l}0.6 \\
0.2\end{array}$ & $\begin{array}{l}0 \\
2.8\end{array}$ \\
\hline Capitella spp. (PO) & $\begin{array}{l}1980 \\
1983\end{array}$ & $\begin{array}{r}316.6 \\
34.4\end{array}$ & $\begin{array}{c}579.2 \\
\text { nd }\end{array}$ & $\begin{array}{r}172.6 \\
0.2\end{array}$ & $\begin{array}{l}4.2 \\
0.8\end{array}$ & $\begin{array}{l}\text { nd } \\
1.0\end{array}$ & $\begin{array}{l}9.6 \\
0.8\end{array}$ & $\begin{array}{c}\text { nd } \\
0\end{array}$ & $\begin{array}{l}0.2 \\
0\end{array}$ & $\begin{array}{l}0.2 \\
1.0\end{array}$ \\
\hline Euphilomedes spp. (OS) & $\begin{array}{l}1980 \\
1983\end{array}$ & $\begin{array}{r}0.2 \\
84.6\end{array}$ & $\begin{array}{c}0,4 \\
\text { nd }\end{array}$ & $\begin{array}{r}0.2 \\
19.6\end{array}$ & $\begin{array}{r}0.6 \\
35.8\end{array}$ & $\begin{array}{l}\text { nd } \\
48.6\end{array}$ & $\begin{array}{l}10.2 \\
54.2\end{array}$ & $\begin{array}{c}\text { nd } \\
107.6\end{array}$ & $\begin{array}{r}74.2 \\
3.0\end{array}$ & $\begin{array}{l}2.2 \\
1.2\end{array}$ \\
\hline Tharyx sp. A $(\mathrm{PO})$ & $\begin{array}{l}1980 \\
1983\end{array}$ & $\begin{array}{r}0.2 \\
34.6\end{array}$ & $\begin{array}{l}0.6 \\
\text { nd }\end{array}$ & $\begin{array}{r}0.2 \\
46.8\end{array}$ & $\begin{array}{l}42.4 \\
24.4\end{array}$ & $\begin{array}{c}\text { nd } \\
268.6\end{array}$ & $\begin{array}{r}1194.0 \\
394.0\end{array}$ & $\begin{array}{c}\text { nd } \\
509.6\end{array}$ & $\begin{array}{r}113.8 \\
30.0\end{array}$ & $\begin{array}{l}0.8 \\
1.4\end{array}$ \\
\hline Macoma carlottensis (PE) & $\begin{array}{l}1980 \\
1983\end{array}$ & $\begin{array}{l}2.4 \\
3.4\end{array}$ & $\begin{array}{c}8.8 \\
\text { nd }\end{array}$ & $\begin{array}{r}10.6 \\
3.2\end{array}$ & $\begin{array}{l}0.2 \\
1.2\end{array}$ & $\begin{array}{l}\text { nd } \\
2.0\end{array}$ & $\begin{array}{l}4.2 \\
2.8\end{array}$ & $\begin{array}{l}\text { nd } \\
1.0\end{array}$ & $\begin{array}{l}0.4 \\
0.4\end{array}$ & $\begin{array}{l}0 \\
0.4\end{array}$ \\
\hline Nereis procera $(\mathrm{PO})$ & $\begin{array}{l}1980 \\
1983\end{array}$ & $\begin{array}{c}10.6 \\
0\end{array}$ & $\begin{array}{l}6.0 \\
\text { nd }\end{array}$ & $\begin{array}{r}11.4 \\
0.8\end{array}$ & $\begin{array}{l}5.2 \\
2.6\end{array}$ & $\begin{array}{l}\text { nd } \\
1.4\end{array}$ & $\begin{array}{r}26.8 \\
5.2\end{array}$ & $\begin{array}{l}\text { nd } \\
5.8\end{array}$ & $\begin{array}{l}6.4 \\
2.4\end{array}$ & $\begin{array}{l}0.2 \\
0.8\end{array}$ \\
\hline Notomastus sp. A (PO) & $\begin{array}{l}1980 \\
1983\end{array}$ & $\begin{array}{l}0 \\
0.6\end{array}$ & $\begin{array}{c}0 \\
\text { nd }\end{array}$ & $\begin{array}{l}0.2 \\
4.6\end{array}$ & $\begin{array}{l}17.8 \\
15.8\end{array}$ & $\begin{array}{l}\text { nd } \\
9.8\end{array}$ & $\begin{array}{l}27.6 \\
19.4\end{array}$ & $\begin{array}{l}\text { nd } \\
15.0\end{array}$ & $\begin{array}{l}2.6 \\
1.4\end{array}$ & $\begin{array}{l}0 \\
0.2\end{array}$ \\
\hline Parvilucina tenuisculpta (PE) & $\begin{array}{l}1980 \\
1983\end{array}$ & $\begin{array}{r}3.6 \\
15.0\end{array}$ & $\begin{array}{l}2.6 \\
\text { nd }\end{array}$ & $\begin{array}{l}12.2 \\
27.4\end{array}$ & $\begin{array}{r}335.4 \\
98.6\end{array}$ & $\begin{array}{c}\text { nd } \\
384.0\end{array}$ & $\begin{array}{r}1498.6 \\
807.6\end{array}$ & $\begin{array}{c}\text { nd } \\
270.4\end{array}$ & $\begin{array}{r}155.0 \\
30.6\end{array}$ & $\begin{array}{r}33.4 \\
3.2\end{array}$ \\
\hline Listriolobus pelodes $(\mathrm{E})$ & $\begin{array}{l}1980 \\
1983\end{array}$ & $\begin{array}{l}0 \\
1.0\end{array}$ & $\begin{array}{c}0 \\
\text { nd }\end{array}$ & $\begin{array}{r}28.6 \\
2.0\end{array}$ & $\begin{array}{l}6.0 \\
0.8\end{array}$ & $\begin{array}{l}\mathrm{nd} \\
1.8\end{array}$ & $\begin{array}{l}45.2 \\
11.0\end{array}$ & $\begin{array}{l}\text { nd } \\
5.0\end{array}$ & $\begin{array}{r}24.4 \\
2.2\end{array}$ & $\begin{array}{l}0.8 \\
0\end{array}$ \\
\hline Tubulanus spp. (N) & $\begin{array}{l}1980 \\
1983\end{array}$ & $\begin{array}{l}0 \\
2.6\end{array}$ & $\begin{array}{c}0.2 \\
\text { nd }\end{array}$ & $\begin{array}{l}0.6 \\
1.8\end{array}$ & $\begin{array}{r}15.2 \\
4.4\end{array}$ & $\begin{array}{l}\text { nd } \\
14.8\end{array}$ & $\begin{array}{l}56.8 \\
19.2\end{array}$ & $\begin{array}{l}\text { nd } \\
12.2\end{array}$ & $\begin{array}{r}12.2 \\
8.4\end{array}$ & $\begin{array}{l}0.6 \\
3.6\end{array}$ \\
\hline Mediomastus californiensis (PO) & $\begin{array}{l}1980 \\
1983\end{array}$ & $\begin{array}{c}0 \\
26.6\end{array}$ & $\begin{array}{l}0.2 \\
\text { nd }\end{array}$ & $\begin{array}{r}0.2 \\
21.2\end{array}$ & $\begin{array}{l}83.8 \\
52.0\end{array}$ & $\begin{array}{c}\text { nd } \\
128.2\end{array}$ & $\begin{array}{r}529.4 \\
47.6\end{array}$ & $\begin{array}{l}\text { nd } \\
41.4\end{array}$ & $\begin{array}{r}81.6 \\
7.4\end{array}$ & $\begin{array}{r}7.6 \\
25.2\end{array}$ \\
\hline Tellina modesta $(\mathrm{PE})$ & $\begin{array}{l}1980 \\
1983\end{array}$ & $\begin{array}{r}0.6 \\
21.4\end{array}$ & $\begin{array}{l}2.4 \\
\text { nd }\end{array}$ & $\begin{array}{r}2.4 \\
21.8\end{array}$ & $\begin{array}{l}2.2 \\
8.4\end{array}$ & $\begin{array}{l}\text { nd } \\
16.0\end{array}$ & $\begin{array}{r}3.6 \\
11.2\end{array}$ & $\begin{array}{l}\text { nd } \\
8.8\end{array}$ & $\begin{array}{r}44.4 \\
6.6\end{array}$ & $\begin{array}{l}3.8 \\
4.4\end{array}$ \\
\hline Spiophanes missionensis (PO) & $\begin{array}{l}1980 \\
1983\end{array}$ & $\begin{array}{l}0 \\
3.6\end{array}$ & $\begin{array}{c}0 \\
\text { nd }\end{array}$ & $\begin{array}{l}1.8 \\
5.4\end{array}$ & $\begin{array}{r}2.8 \\
15.2\end{array}$ & $\begin{array}{l}\text { nd } \\
28.8\end{array}$ & $\begin{array}{l}67.6 \\
58.4\end{array}$ & $\begin{array}{l}\text { nd } \\
76.8\end{array}$ & $\begin{array}{l}74.2 \\
46.8\end{array}$ & $\begin{array}{l}39.2 \\
42.2\end{array}$ \\
\hline Axinopsida serricata (PE) & $\begin{array}{l}1980 \\
1983\end{array}$ & $\begin{array}{l}0 \\
0.4\end{array}$ & $\begin{array}{c}0 \\
\text { nd }\end{array}$ & $\begin{array}{c}0 \\
10.8\end{array}$ & $\begin{array}{l}0.4 \\
2.0\end{array}$ & $\begin{array}{l}\text { nd } \\
3.4\end{array}$ & $\begin{array}{r}15.8 \\
4.0\end{array}$ & $\begin{array}{l}\text { nd } \\
13.2\end{array}$ & $\begin{array}{l}95.4 \\
20.6\end{array}$ & $\begin{array}{r}21.0 \\
9.6\end{array}$ \\
\hline Pectinaria californiensis (PO) & $\begin{array}{l}1980 \\
1983\end{array}$ & $\begin{array}{l}0 \\
1.0\end{array}$ & $\begin{array}{c}0 \\
\text { nd }\end{array}$ & $\begin{array}{r}1.8 \\
13.4\end{array}$ & $\begin{array}{r}22.0 \\
7.4\end{array}$ & $\begin{array}{l}\text { nd } \\
10.4\end{array}$ & $\begin{array}{l}51.2 \\
14.6\end{array}$ & $\begin{array}{l}\text { nd } \\
19.2\end{array}$ & $\begin{array}{l}52.6 \\
24.4\end{array}$ & $\begin{array}{l}15.2 \\
29.4\end{array}$ \\
\hline Minuspio cirrifera $(\mathrm{PO})$ & $\begin{array}{l}1980 \\
1983\end{array}$ & $\begin{array}{l}0 \\
5.4\end{array}$ & $\begin{array}{c}0 \\
\mathrm{nd}\end{array}$ & $\begin{array}{l}0.2 \\
6.2\end{array}$ & $\begin{array}{l}0.4 \\
0.6\end{array}$ & $\begin{array}{l}\text { nd } \\
0.4\end{array}$ & $\begin{array}{l}0.4 \\
0.8\end{array}$ & $\begin{array}{l}\text { nd } \\
0.2\end{array}$ & $\begin{array}{l}3.8 \\
1.8\end{array}$ & $\begin{array}{r}1.0 \\
46.6\end{array}$ \\
\hline Myriochele sp. M (PO) & $\begin{array}{l}1980 \\
1983\end{array}$ & $\begin{array}{l}0 \\
0\end{array}$ & $\begin{array}{c}0 \\
\text { nd }\end{array}$ & $\begin{array}{l}0 \\
0\end{array}$ & $\begin{array}{l}0 \\
0\end{array}$ & $\begin{array}{c}\mathrm{nd} \\
0\end{array}$ & $\begin{array}{l}0 \\
0\end{array}$ & $\begin{array}{l}\text { nd } \\
0.2\end{array}$ & $\begin{array}{l}0.8 \\
0\end{array}$ & $\begin{array}{r}53.0 \\
1.6\end{array}$ \\
\hline Amphiodia urtica (OP) & $\begin{array}{l}1980 \\
1983\end{array}$ & $\begin{array}{l}0 \\
0\end{array}$ & $\begin{array}{c}0 \\
\text { nd }\end{array}$ & $\begin{array}{l}0 \\
0\end{array}$ & $\begin{array}{l}0 \\
0\end{array}$ & $\begin{array}{c}\text { nd } \\
0\end{array}$ & $\begin{array}{l}0 \\
0.2\end{array}$ & $\begin{array}{c}\text { nd } \\
0\end{array}$ & $\begin{array}{l}0.2 \\
0\end{array}$ & $\begin{array}{r}131.0 \\
89.4\end{array}$ \\
\hline Rhepoxynius bicuspidatus (A) & $\begin{array}{l}1980 \\
1983\end{array}$ & $\begin{array}{l}0 \\
0\end{array}$ & $\begin{array}{c}0 \\
\text { nd }\end{array}$ & $\begin{array}{l}0 \\
0\end{array}$ & $\begin{array}{l}0 \\
0\end{array}$ & $\begin{array}{c}\text { nd } \\
0\end{array}$ & $\begin{array}{l}0 \\
0\end{array}$ & $\begin{array}{c}\text { nd } \\
0\end{array}$ & $\begin{array}{l}0 \\
0\end{array}$ & $\begin{array}{r}23.0 \\
5.4\end{array}$ \\
\hline
\end{tabular}


always less abundant in 1983 than in 1980 at Stns 4, 6, and 8 . The polychaete Spiophanes missionensis and Euphilomedes spp. were usually ranked among the 5 most abundant species at Stns 4 through 8 in 1983.

The brittlestar Amphiodia urtica was the most abundant species at the reference station (9) in 1980 and 1983 (Table 1). A. urtica was rare or absent at all other stations. This is also true of 2 other abundant species at Stn 9 in 1980, the amphipod Rhepoxynius bicuspidatus and the polychaete Myriochele sp. M. However, most of the other dominants at Stn 9 were usually found on the Palos Verdes Shelf. Dominants as close to the outfalls as Stn 3 were sometimes among the 5 most abundant species at $\operatorname{Stn} 9$, e.g. Parvilucina tenuisculpta in 1980 and Mediomastus californiensis in 1983.

Changes in the distribution of dominant species on the Palos Verdes Shelf near the LACSD outfalls between 1980 and 1983 can be classified as follows:

Less abundant near outfalls

Polychaetes

Eumida sanguinea

Schistomeringos longicornis

Capitella spp.

Nereis procera

Closer to or more abundant near outfalls

Polychaetes

Tharyx sp. A

Notomastus sp. A

Mediomastus californiensis

Spiophanes missionensis

Pectinaria californiensis

Minuspio cirrifera

Bivalves

Parvilucina tenuisculpta

Tellina modesta

Axinopsida semicata

Ostracods

Euphilomedes spp.

\section{Community structure}

Macrobenthos species richness, biomass, and density increased above background levels on the Palos Verdes Shelf in both 1980 and 1983 (Fig. 2). In both years the maximum values for these parameters occurred at Stn 6 , about $9 \mathrm{~km}$ from the outfalls, and then decreased progressively at stations closer to the outfalls. Although this between-station spatial pattern was similar in both years, there were significant changes between 1980 and 1983 in the structure of the benthos at most stations (Table 2). At Stn 1 there were
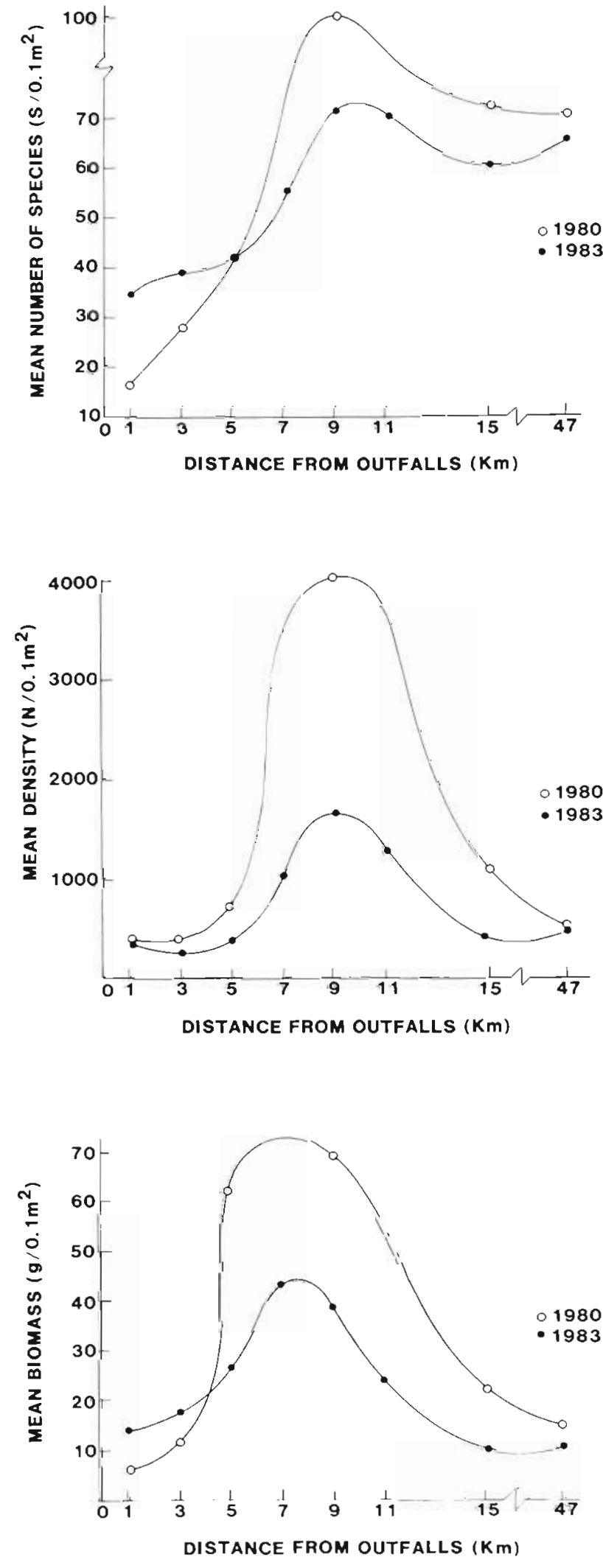

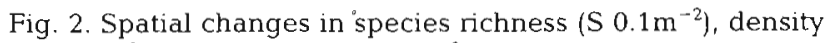
( $\mathrm{N} 0.1 \mathrm{~m}^{-2}$ ), and biomass (g $0.1 \mathrm{~m}^{-2}$ ), of the macrobenthos near the Los Angeles County sewage outfalls in 1980 and 1983 
significant increases in 1983 in species richness, biomass, and the Infaunal Index. Numerical dominance decreased significantly and amphipods were present at Stn 1 in 1983, but not in 1980. Macrobenthos density in 1983 at Stn 1 was not significantly different from 1980. Changes between years at Stn 3 were similar to those at Stn 1; there were significantly more species, higher Infaunal Index, and lower numerical dominance. Biomass at Stn 3 increased by $79 \%$ in 1983 , but the difference from 1980 was not significant.

The greatest quantitative changes in the benthos of the Palos Verdes Shelf occurred at Stns 4, 6, and 8 where density and biomass decreased significantly between 1980 and 1983 (Table 2). Infaunal Index increased significantly in 1983 at Stns 4 and 8, but did not change at Stn 6. Phoxocephalid amphipods were present at Stn 4 in 1983, but not in 1980. The abundance of phoxocephalids and all amphipods decreased significantly at Stns 6 and 8 in 1983 .

The benthos changed less at the reference station (9) than at any other station. There were no significant differences there between 1980 and 1983 in density, species richness, dominance, Infaunal Index, or biomass (Table 2). However, phoxocephalid amphipods and other amphipod densities declined in 1983.

Faunal homogeneity within stations was consistently high relative to homogeneity between stations or between years. Numerical classification of the macrobenthos data always placed replicate grab samples from the same station in the same year within the same cluster (Fig. 3). The dendrogram reflects strong spatial and temporal biological gradients. Larger station clusters are evident in Fig. 3. Replicates within these large clusters were pooled for statistical comparisons of biological conditions (Table 3). Stns 1, 2, and 3 in 1980 form a distinct cluster at one extreme in the biological gradient. All samples from Stn 9 in both 1980 and 1983 group to form a cluster representing background conditions at the other extreme of the gradient. The background assemblage had the highest Infaunal Index of any cluster, relatively low biomass and density of individuals, and very low numerical dominance. Echinoderms and amphipods were abundant. The most abundant species was the brittlestar Amphiodia urtica. Two clusters in Fig. 3 are associated with an apparent

Table 2. Structural comparison of the macrobenthic community on the Palos Verdes Shelf and Santa Monica Bay, California in 1980 and 1983. Data are mean values for 5 replicate $0.1 \mathrm{~m}^{2}$ grabs taken at each station

\begin{tabular}{|c|c|c|c|c|c|c|c|}
\hline \multirow[t]{2}{*}{ Variable } & \multirow[t]{2}{*}{ Year } & \multicolumn{6}{|c|}{ Station } \\
\hline & & 1 & 3 & 4 & 6 & 8 & 9 \\
\hline $\begin{array}{l}\text { Density } \\
\left(\mathrm{N} 0.1 \mathrm{~m}^{-2}\right)\end{array}$ & $\begin{array}{l}1980 \\
1983\end{array}$ & $\begin{array}{c}371.8 \\
320.0 \\
\text { NS }\end{array}$ & $\begin{array}{c}307.0 \\
292.7 \\
\text { NS }\end{array}$ & $\begin{array}{l}720.2 \\
334.2 \\
-\cdots\end{array}$ & $\begin{array}{c}4086.0 \\
1656.2 \\
-\cdots\end{array}$ & $\begin{array}{r}1082.0 \\
354.6 \\
-\quad \cdots\end{array}$ & $\begin{array}{c}528.6 \\
489.6 \\
\text { NS }\end{array}$ \\
\hline $\begin{array}{l}\text { Species richness } \\
\left(\mathrm{S} 0.1 \mathrm{~m}^{-2}\right)\end{array}$ & $\begin{array}{l}1980 \\
1983\end{array}$ & $\begin{array}{r}15.8 \\
34.8 \\
+\cdots\end{array}$ & $\begin{array}{r}28.2 \\
39.3 \\
+\quad "\end{array}$ & $\begin{array}{l}41.2 \\
41.2 \\
\text { NS }\end{array}$ & $\begin{array}{l}96.2 \\
70.4 \\
-\cdot\end{array}$ & $\begin{array}{l}71.8 \\
59.8 \\
-\end{array}$ & $\begin{array}{l}70.4 \\
66.6 \\
\text { NS }\end{array}$ \\
\hline $\begin{array}{l}\text { Dominance index } \\
\left(\mathrm{S} 75 \% \mathrm{~N}^{-1}\right)\end{array}$ & $\begin{array}{l}1980 \\
1983\end{array}$ & $\begin{array}{r}1.02 \\
7.30 \\
+\cdots\end{array}$ & $\begin{array}{r}4.6 \\
9.6 \\
+\quad \cdot\end{array}$ & $\begin{array}{r}2.8 \\
6.0 \\
+\cdots\end{array}$ & $\begin{array}{r}2.6 \\
3.1 \\
\text { NS }\end{array}$ & $\begin{array}{r}10.8 \\
16.6 \\
+\cdots\end{array}$ & $\begin{array}{l}11.8 \\
13.8 \\
\text { NS }\end{array}$ \\
\hline $\begin{array}{l}\text { Amphipod density } \\
\left(\mathrm{N} 0.1 \mathrm{~m}^{-2}\right)\end{array}$ & $\begin{array}{l}1980 \\
1983\end{array}$ & $\begin{array}{r}0.0 \\
9.4 \\
+\cdots\end{array}$ & $\begin{array}{c}2.4 \\
4.3 \\
\text { NS }\end{array}$ & $\begin{array}{c}1.6 \\
2.2 \\
\text { NS }\end{array}$ & $\begin{array}{l}46.0 \\
21.2 \\
-\end{array}$ & $\begin{array}{r}52.2 \\
20.8 \\
-\cdots\end{array}$ & $\begin{array}{r}65.4 \\
20.8 \\
-\quad \cdots\end{array}$ \\
\hline $\begin{array}{l}\text { Phoxocephalid density } \\
\text { (N } 0.1 \mathrm{~m}^{-2} \text { ) }\end{array}$ & $\begin{array}{l}1980 \\
1983\end{array}$ & $\begin{array}{c}0.0 \\
0.0 \\
\text { NS }\end{array}$ & $\begin{array}{c}0.0 \\
0.0 \\
\text { NS }\end{array}$ & $\begin{array}{c}0.0 \\
0.4 \\
\text { NS }\end{array}$ & $\begin{array}{r}30.6 \\
11.6 \\
-\cdots\end{array}$ & $\begin{array}{r}40.8 \\
12.6 \\
-\cdots\end{array}$ & $\begin{array}{r}31.4 \\
9.4 \\
-\cdots\end{array}$ \\
\hline $\begin{array}{l}\text { Echinoderm density } \\
\text { (N } 0.1 \mathrm{~m}^{-2} \text { ) }\end{array}$ & $\begin{array}{l}1980 \\
1983\end{array}$ & $\begin{array}{c}0.0 \\
0.2 \\
\text { NS }\end{array}$ & $\begin{array}{c}0.2 \\
0.2 \\
\text { NS }\end{array}$ & $\begin{array}{c}0.0 \\
0.0 \\
\text { NS }\end{array}$ & $\begin{array}{r}0.0 \\
0.4 \\
\text { NS }\end{array}$ & $\begin{array}{r}0.2 \\
0.2 \\
\text { NS }\end{array}$ & $\begin{array}{c}136.0 \\
93.4 \\
\text { NS }\end{array}$ \\
\hline Infaunal Index & $\begin{array}{l}1980 \\
1983\end{array}$ & $\begin{array}{r}4.5 \\
52.8 \\
+\cdots\end{array}$ & $\begin{array}{r}18.4 \\
58.6 \\
+\cdots\end{array}$ & $\begin{array}{r}37.1 \\
52.8 \\
+\cdots\end{array}$ & $\begin{array}{l}51.9 \\
50.6 \\
\text { NS }\end{array}$ & $\begin{array}{r}63.5 \\
68.3 \\
+\quad .\end{array}$ & $\begin{array}{l}81.0 \\
79.6 \\
\text { NS }\end{array}$ \\
\hline $\begin{array}{l}\text { Biomass } \\
\left(\mathrm{g} 0.1 \mathrm{~m}^{-2}\right)\end{array}$ & $\begin{array}{l}1980 \\
1983\end{array}$ & $\begin{array}{r}6.5 \\
14.3 \\
+\cdot\end{array}$ & $\begin{array}{l}9.9 \\
17.7 \\
\text { NS }\end{array}$ & $\begin{array}{r}61.6 \\
27.2 \\
-\quad \cdots\end{array}$ & $\begin{array}{r}68.7 \\
39.4 \\
-\cdots\end{array}$ & $\begin{array}{r}20.8 \\
10.2 \\
-\cdot\end{array}$ & $\begin{array}{l}14.4 \\
11.5 \\
\text { NS }\end{array}$ \\
\hline
\end{tabular}




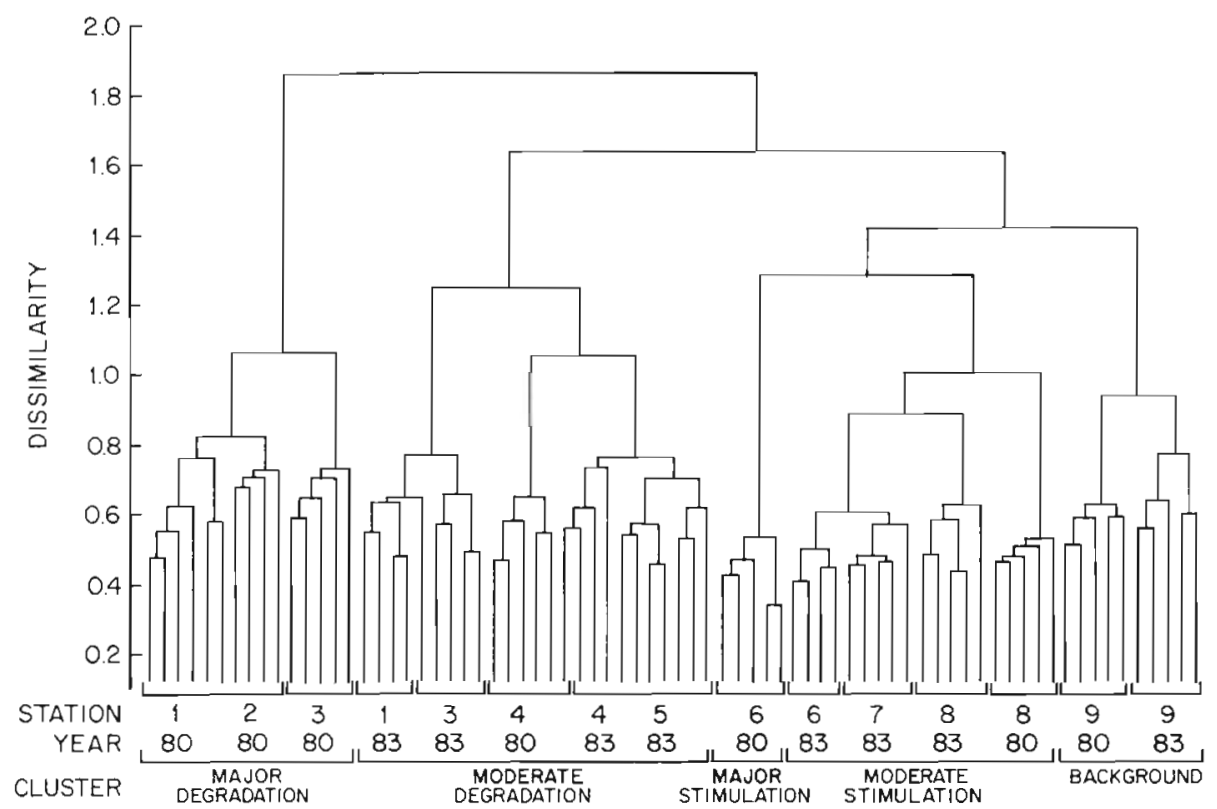

Fig. 3. Numerical classification of macrobenthos samples. Primary clusters include replicate grab samples from each station/ year combination. Description of secondary clusters is based on faunal changes (Table 3) stimulation of the benthos as reflected by significant increases in density or species richness from the background cluster mean. The 'moderate stimulation' cluster includes samples from Stns 6, 7, and 8 in 1983 and Stn 8 in 1980. The dominant species in this cluster was Parvilucina tenuisculpta. Amphipods were abundant, but echinoderms were rare. Species richness was not significantly different from the reference assemblage, but density of individuals was twice as great. Replicates from Stn 6 in 1980 form a unique cluster of major benthic stimulation because there were significantly more species, biomass, and individuals than in the reference assemblage. In the 2 clusters associated with a degradation of the benthos there were fewer species and individuals and lower biomass than in the condition of stimulation. Echinoderms and amphipods were rare. Samples from Stns 1, 3, 4, and 5 in 1983 and 4 in 1980 are included in the cluster of moderate degradation. The major degradation at Stns 1, 2, and 3 in 1980 is different from moderate degradation because of the greater dominance of Capitella spp. and significantly lower values of Infaunal Index, species richness, and biomass.

Both spatial and temporal gradients are evident in the numerical classification (Fig. 3). For either year the hierarchial arrangement of macrobenthic sample clus-

Table 3. Structural characteristics of macrobenthic communities at station clusters on the Palos Verdes Shelf in 1980 and 1983 Station clusters are defined by numerical classification (Fig. 3). All data are mean values per $0.1 \mathrm{~m}^{2}$ for all grab samples pooled within each station cluster. Means followed by different letters within the same column are significantly different ( $p<0.05)$. (The letters have no purpose other than to indicate the results of the multiple range test)

\begin{tabular}{|c|c|c|c|c|c|c|c|c|c|}
\hline $\begin{array}{l}\text { Station } \\
\text { cluster }\end{array}$ & $\begin{array}{l}\text { Year: } \\
\text { stations }\end{array}$ & $\begin{array}{c}\text { Number of } \\
\text { species }\end{array}$ & $\begin{array}{l}\text { Number of } \\
\text { individuals }\end{array}$ & $\begin{array}{l}\text { Number of } \\
\text { amphipods }\end{array}$ & $\begin{array}{l}\text { Number of } \\
\text { echinoderms }\end{array}$ & $\begin{array}{l}\text { Biomass } \\
\text { (g) }\end{array}$ & $\begin{array}{l}\text { Dominance } \\
\text { index }\end{array}$ & $\begin{array}{l}\text { Infaunal } \\
\text { Index }\end{array}$ & Dominant species \\
\hline $\begin{array}{l}\text { Major } \\
\text { degradation }\end{array}$ & $1980: 1,2,3$ & $20.2^{\mathrm{D}}$ & $447^{\mathrm{A}}$ & $0.8^{C}$ & $0.1^{\mathrm{B}}$ & $9.4^{\mathrm{A}}$ & $2.2^{\mathrm{C}}$ & $8.5^{\mathrm{D}}$ & Capitella capitata \\
\hline $\begin{array}{l}\text { Moderate } \\
\text { degradation }\end{array}$ & $\begin{array}{l}1980: 4 \\
1983: 1,3,4,5\end{array}$ & $42.8^{\mathrm{C}}$ & $566^{\mathrm{A}}$ & $4.4^{\mathrm{B}}$ & $0.1^{\mathrm{B}}$ & $34.2^{\mathrm{C}}$ & $5.4^{8}$ & $50.3^{C}$ & Parvilucina tenusisculpta \\
\hline $\begin{array}{l}\text { Major } \\
\text { stimulation }\end{array}$ & 1980: 6 & $96.2^{B}$ & $4086^{C}$ & $46.0^{A}$ & $0.0^{\mathrm{B}}$ & $68.7^{\mathrm{D}}$ & $2.6^{\mathrm{C}}$ & $51.9^{c}$ & Parvilucina tenuisculpta \\
\hline $\begin{array}{l}\text { Moderate } \\
\text { stimulation }\end{array}$ & $\begin{array}{l}1980: 8 \\
1983: 6,7,8\end{array}$ & $67.8^{A}$ & $1095^{\mathrm{B}}$ & $31.4^{\mathrm{A}}$ & $0.3^{\mathrm{B}}$ & $23.6^{\mathrm{BC}}$ & $8.6^{\mathrm{AB}}$ & $60.6^{\mathrm{B}}$ & Parvilucina tenuisclilpta \\
\hline Background & $\begin{array}{l}\text { 1980: } 9 \\
1983: 9\end{array}$ & $68.5^{\mathrm{A}}$ & $509^{A}$ & $43.1^{\mathrm{A}}$ & $114.7^{A}$ & $13.4^{\mathrm{AB}}$ & $12.8^{\mathrm{A}}$ & $80.3^{\mathrm{A}}$ & Amphiodia urtica \\
\hline \multicolumn{2}{|c|}{ Statistical comparison } & $\begin{array}{c}F=20.9 \\
p<0.001\end{array}$ & $\begin{array}{l}F=110.7 \\
p<0.001\end{array}$ & $\begin{array}{c}F=74.2 \\
p<0.001\end{array}$ & $\begin{array}{l}F=640.7 \\
p<0.001\end{array}$ & $\begin{array}{l}F=17.2 \\
p<0.001\end{array}$ & $\begin{array}{l}H=38.4 \\
p<0.001\end{array}$ & $\begin{array}{l}F=150.1 \\
p<0.001\end{array}$ & \\
\hline
\end{tabular}


Table 4. Chemical characteristics of sediment from the Palos Verdes Shelf and Santa Monica Bay, California in 1980 and 1983. All bulk sediment concentrations are expressed on a dry weight basis. Data are mean values for 5 replicate grabs taken at each station in each year

\begin{tabular}{|c|c|c|c|c|c|c|c|}
\hline \multirow[t]{2}{*}{ Parameter } & \multirow{2}{*}{ Year } & \multicolumn{6}{|c|}{ Station } \\
\hline & & 1 & 3 & 4 & 6 & 8 & 9 \\
\hline $\mathrm{Eh}, \mathrm{mV}$ & $\begin{array}{l}1980 \\
1983\end{array}$ & $\begin{array}{r}-85 \\
44 \\
\text { NS }\end{array}$ & $\begin{array}{r}-29 \\
132 \\
+\cdots\end{array}$ & $\begin{array}{r}89 \\
210 \\
\text { NS }\end{array}$ & $\begin{array}{l}219 \\
114 \\
\text { NS }\end{array}$ & $\begin{array}{l}363 \\
310 \\
\text { NS }\end{array}$ & $\begin{array}{l}321 \\
251 \\
\text { NS }\end{array}$ \\
\hline Total volatile solids, $\%$ & $\begin{array}{l}1980 \\
1983\end{array}$ & $\begin{array}{r}17.5 \\
12.1 \\
-\cdots\end{array}$ & $\begin{array}{l}15.0 \\
11.4 \\
-\end{array}$ & $\begin{array}{l}14.2 \\
11.8 \\
-\end{array}$ & $\begin{array}{r}15.2 \\
10.6 \\
-\cdots\end{array}$ & $\begin{array}{r}9.5 \\
6.9 \\
-\cdots\end{array}$ & $\begin{array}{l}4.0 \\
6.6 \\
\text { NS }\end{array}$ \\
\hline Total organic carbon, \% & $\begin{array}{l}1980 \\
1983\end{array}$ & $\begin{array}{l}4.1 \\
3.1 \\
-\end{array}$ & $\begin{array}{l}3.7 \\
3.3 \\
\text { NS }\end{array}$ & $\begin{array}{l}3.8 \\
3.0 \\
\text { NS }\end{array}$ & $\begin{array}{l}3.2 \\
2.4 \\
-\end{array}$ & $\begin{array}{l}2.2 \\
1.4 \\
-\end{array}$ & $\begin{array}{l}0.9 \\
1.3 \\
\text { NS }\end{array}$ \\
\hline Ammonia- $N$, mg $\mathrm{l}^{-1}$ & $\begin{array}{l}1980 \\
1983\end{array}$ & $\begin{array}{l}2.4 \\
2.0 \\
\text { NS }\end{array}$ & $\begin{array}{l}1.4 \\
1.5 \\
\text { NS }\end{array}$ & $\begin{array}{l}0.40 \\
1.3 \\
+\end{array}$ & $\begin{array}{l}0.34 \\
0.50 \\
\text { NS }\end{array}$ & $\begin{array}{l}0.56 \\
0.53 \\
\text { NS }\end{array}$ & $\begin{array}{l}0.44 \\
1.6 \\
\text { NS }\end{array}$ \\
\hline Free sulfide, $\mathrm{mg} \mathrm{l}^{-1}$ & $\begin{array}{l}1980 \\
1983\end{array}$ & $\begin{array}{r}11 \\
1.2 \\
-\cdots\end{array}$ & $\begin{array}{l}6.7 \\
1.5 \\
\text { NS }\end{array}$ & $\begin{array}{c}0.49 \\
0.37 \\
\text { NS }\end{array}$ & $\begin{array}{l}0.65 \\
0.27 \\
-\end{array}$ & $\begin{array}{l}0.41 \\
1.2 \\
\text { NS }\end{array}$ & $\begin{array}{l}0.33 \\
0.37 \\
\text { NS }\end{array}$ \\
\hline Total sulfide, $\mathrm{mg} \mathrm{kg}^{-1}$ & $\begin{array}{l}1980 \\
1983\end{array}$ & $\begin{array}{c}2520 \\
1838 \\
\text { NS }\end{array}$ & $\begin{array}{c}2370 \\
1349 \\
-{ }^{-}\end{array}$ & $\begin{array}{c}1310 \\
1532 \\
\text { NS }\end{array}$ & $\begin{array}{l}443 \\
572 \\
\text { NS }\end{array}$ & $\begin{array}{l}121 \\
157 \\
\text { NS }\end{array}$ & $\begin{array}{r}24 \\
226 \\
\text { NS }\end{array}$ \\
\hline $5 \mathrm{~d} \mathrm{BOD}, \mathrm{mg} \mathrm{kg}{ }^{-1}$ & $\begin{array}{l}1980 \\
1983\end{array}$ & $\begin{array}{r}13800 \\
3990 \\
-\cdots\end{array}$ & $\begin{array}{r}10600 \\
4540 \\
-\cdots\end{array}$ & $\begin{array}{r}7160 \\
3250 \\
-\cdots\end{array}$ & $\begin{array}{l}4390 \\
2100 \\
-\cdots\end{array}$ & $\begin{array}{c}1740 \\
880 \\
-\cdot\end{array}$ & $\begin{array}{l}660 \\
680 \\
\text { NS }\end{array}$ \\
\hline Total oil/grease, $\mathrm{mg} \mathrm{kg}^{-1}$ & $\begin{array}{l}1980 \\
1983\end{array}$ & $\begin{array}{r}20600 \\
6860 \\
-\cdots\end{array}$ & $\begin{array}{c}9950 \\
5280 \\
-\end{array}$ & $\begin{array}{c}6180 \\
5060 \\
\text { NS }\end{array}$ & $\begin{array}{r}3620 \\
1620 \\
-\cdots\end{array}$ & $\begin{array}{c}1020 \\
610 \\
-\cdot\end{array}$ & $\begin{array}{l}338 \\
516 \\
\text { NS }\end{array}$ \\
\hline $\begin{array}{l}\text { Hydrocarbon oil/grease, } \\
\mathrm{mg} \mathrm{kg}^{-1}\end{array}$ & $\begin{array}{l}1980 \\
1983\end{array}$ & $\begin{array}{r}10800 \\
6180 \\
-\cdots\end{array}$ & $\begin{array}{c}7020 \\
4500 \\
\text { NS }\end{array}$ & $\begin{array}{c}4920 \\
4590 \\
\text { NS }\end{array}$ & $\begin{array}{l}2270 \\
1290 \\
-\cdots\end{array}$ & $\begin{array}{r}645 \\
503 \\
-\cdots\end{array}$ & $\begin{array}{l}144 \\
291 \\
\text { NS }\end{array}$ \\
\hline Cadmium, mg kg ${ }^{-1}$ & $\begin{array}{l}1980 \\
1983\end{array}$ & $\begin{array}{c}32 \\
26 \\
\text { NS }\end{array}$ & $\begin{array}{c}26 \\
14 \\
-\end{array}$ & $\begin{array}{c}22 \\
18 \\
\text { NS }\end{array}$ & $\begin{array}{r}8.9 \\
4.5 \\
-\cdots\end{array}$ & $\begin{array}{r}2.4 \\
1.8 \\
-\end{array}$ & $\begin{array}{l}2.4 \\
0.7 \\
\text { NS }\end{array}$ \\
\hline Lead, $\mathrm{mg} \mathrm{kg}^{-1}$ & $\begin{array}{l}1980 \\
1983\end{array}$ & $\begin{array}{r}338 \\
193 \\
-\cdots\end{array}$ & $\begin{array}{l}293 \\
140 \\
-\end{array}$ & $\begin{array}{r}252 \\
147 \\
-\cdots\end{array}$ & $\begin{array}{r}150 \\
45 \\
-\cdots\end{array}$ & $\begin{array}{r}57 \\
31 \\
-\cdots\end{array}$ & $\begin{array}{c}33 \\
47 \\
\text { NS }\end{array}$ \\
\hline Zinc, $\mathrm{mg} \mathrm{kg} \mathrm{kg}^{-1}$ & $\begin{array}{l}1980 \\
1983\end{array}$ & $\begin{array}{l}853 \\
873 \\
\text { NS }\end{array}$ & $\begin{array}{l}584 \\
531 \\
\text { NS }\end{array}$ & $\begin{array}{l}709 \\
577 \\
\text { NS }\end{array}$ & $\begin{array}{r}339 \\
171 \\
-\cdots\end{array}$ & $\begin{array}{l}130 \\
107 \\
\text { NS }\end{array}$ & $\begin{array}{r}95 \\
129 \\
\text { NS }\end{array}$ \\
\hline Chromium, mg kg ${ }^{-1}$ & $\begin{array}{l}1980 \\
1983\end{array}$ & $\begin{array}{c}821 \\
664 \\
-\cdots\end{array}$ & $\begin{array}{l}481 \\
439 \\
\text { NS }\end{array}$ & $\begin{array}{l}720 \\
478 \\
\text { NS }\end{array}$ & $\begin{array}{l}305 \\
180 \\
-\end{array}$ & $\begin{array}{l}101 \\
152 \\
+\end{array}$ & $\begin{array}{c}62 \\
94 \\
\text { NS }\end{array}$ \\
\hline Copper, mg kg ${ }^{-1}$ & $\begin{array}{l}1980 \\
1983\end{array}$ & $\begin{array}{r}691 \\
328 \\
-\cdots\end{array}$ & $\begin{array}{l}450 \\
235 \\
-\end{array}$ & $\begin{array}{r}547 \\
248 \\
-\cdots\end{array}$ & $\begin{array}{r}309 \\
74 \\
-\cdots\end{array}$ & $\begin{array}{r}96 \\
51 \\
-\cdots\end{array}$ & $\begin{array}{r}52 \\
45 \\
\text { NS }\end{array}$ \\
\hline Nickel, mg kg ${ }^{-1}$ & $\begin{array}{l}1980 \\
1983\end{array}$ & $\begin{array}{r}95 \\
76 \\
-\cdots\end{array}$ & $\begin{array}{r}99 \\
63 \\
-\cdots\end{array}$ & $\begin{array}{c}85 \\
70 \\
\text { NS }\end{array}$ & $\begin{array}{r}61 \\
42 \\
-\cdots\end{array}$ & $\begin{array}{l}26 \\
29 \\
\text { NS }\end{array}$ & $\begin{array}{c}61 \\
42 \\
\text { NS }\end{array}$ \\
\hline $\begin{array}{l}\text { Bis (2-ethylhexyl) } \\
\text { phthalate, } \mu g \mathrm{~kg}^{-1}\end{array}$ & $\begin{array}{l}1980^{\circ} \\
1983\end{array}$ & $\begin{array}{c}12730 \\
13960 \\
\text { NS }\end{array}$ & $\begin{array}{c}8800 \\
8460 \\
\text { NS }\end{array}$ & $\begin{array}{c}7200 \\
9310 \\
\text { NS }\end{array}$ & $\begin{array}{l}5120 \\
1820 \\
-\cdots\end{array}$ & $\begin{array}{c}1010 \\
450 \\
\text { NS }\end{array}$ & $\begin{array}{l}140 \\
440 \\
\text { NS }\end{array}$ \\
\hline $4,4^{\prime}-\mathrm{DDE}, \mu \mathrm{g} \mathrm{kg}{ }^{-1}$ & $\begin{array}{l}1980^{\circ} \\
1983\end{array}$ & $\begin{array}{c}5610 \\
6990 \\
\text { NS }\end{array}$ & $\begin{array}{l}5920 \\
4970 \\
\text { NS }\end{array}$ & $\begin{array}{l}7830 \\
7510 \\
\text { NS }\end{array}$ & $\begin{array}{c}1460 \\
2790 \\
\text { NS }\end{array}$ & $\begin{array}{c}970 \\
1050 \\
\text { NS }\end{array}$ & $\begin{array}{r}60 \\
110 \\
\text { NS }\end{array}$ \\
\hline $\begin{array}{l}\text { Number of parameters dec } \\
\text { between } 1980 \text { and } 1983\end{array}$ & sing & 14 & 16 & 14 & 13 & 11 & 3 \\
\hline $\begin{array}{l}\text { 'Only } 2 \text { replicates were an } \\
\text { NS: not significant }- \text { dect } \\
\text { p }<0.05 ; \quad \cdots p<0.01_{i}\end{array}$ & $\begin{array}{l}\text { zed for I } \\
\text { e betwee } \\
<0.001\end{array}$ & $\begin{array}{l}\text { d phth } \\
s_{i}+\text { ir }\end{array}$ & $\begin{array}{l}1980 \\
\text { etwee }\end{array}$ & & & & \\
\hline
\end{tabular}


ters is consistent with a hypothesis of a spatial biological gradient from the stations closest to the outfalls to the reference station. Temporal changes between 1980 and 1983 are shown by shifts in the location of stations from one cluster to another. Stns 1 and 3 moved from the major degradation cluster in 1980 to the moderate degradation cluster in 1983. Stn 6 moved from the major stimulation cluster in 1980 to the moderate stimulation cluster in 1983. Samples from Stns 8 and 9 farthest from the outfalls remained in the same clusters (moderate stimulation and background, respectively) in 1980 and 1983.

The 'background' and 'major degradation' clusters represent the extremes within this data set of biological conditions on the spatial-temporal pollution gradient associated with the LACSD outfalls. The 2 clusters differ greatly in species composition, species richness, dominance, Infaunal Index, and the presence/absence of indicator taxa like amphipods, echinoderms, and Capitella spp. (Table 3). Despite these qualitative differences, there are no significant differences between these clusters in the density or biomass of the benthos.

\section{Sediment contamination}

The concentration of most sediment contaminants declined significantly on the Palos Verdes Shelf between 1980 and 1983 (Table 4). Among 85 temporal comparisons of 17 contaminants at 5 stations on the Shelf, 44 showed significant decreases in sediment concentration. There were only 2 instances in which sediment concentrations increased significantly: ammonia-N at Stn 4 and chromium at Stn 8. (The sig- nificant increase in Eh at Stn 3 is evidence of decreasing sediment organic enrichment.) Four parameters decreased significantly at every station on the Shelf: total volatile solids, sediment BOD, lead, and copper. In contrast, there were no significant changes in sediment concentrations of any parameter at the reference Stn 9 in Santa Monica Bay.

The numerical classification of the chemical data set produced a dendrogram similar to that for the macrobenthos (Fig. 4). Spatial gradients in sediment contamination are evident in the arrangement of clusters from A, containing the most contaminated samples from Stns 1, 2, and 3 in 1980, to $F$, containing most samples from the least contaminated, reference Stn 9. Temporal changes in sediment chemistry are indicated by the complete separation of replicates from Stns 1, 3, and 6 into different clusters depending on the year of collection. In 1983 all replicates from Stns 1, 3, and 6 were placed in clusters representing less contaminated sediment conditions than in 1980. Within-station heterogeneity in sediment characteristics in 1983 is evident in the appearance of replicates from Stns 3, 4, and 9 in adjacent clusters. The heterogeneity at Stn 9 in 1983 may be due to the presence in some grabs of large quantities of terrestrial plant leaves and twigs. This material was not found at any other station.

\section{Sediment toxicity}

Mean survival of Rhepoxynius abronius was significantly less than the Yaquina Bay sediment in 1980 at Stns 1, 2, and 3 (Table 5). All other sediment samples

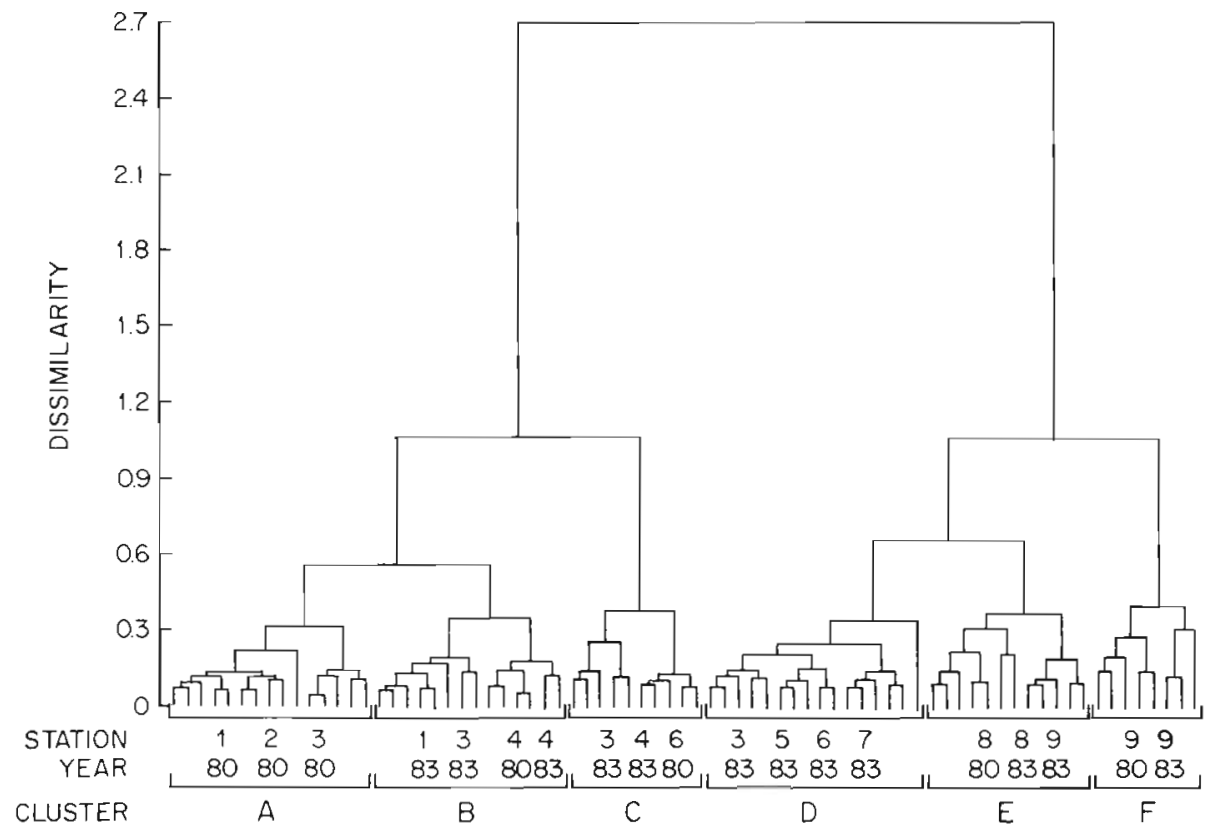

Fig. 4. Numerical classification of sediment chemistry samples. Alphabetically-coded clusters include one or more replicate grab samples from each station year combination 
Table 5. Rhepoxynius abronius. Mean survival in sediment from the Palos Verdes Shelf and Santa Monica Bay, California Twenty amphipods were added to 5 replicate sediment samples from each station in each year. Means underlined are not significantly different from survival in Yaquina Bay, Oregon reference sediment $(\mathrm{p}<0.05)$

\begin{tabular}{|c|c|c|c|c|c|c|c|c|c|c|}
\hline Year & 1 & 2 & 3 & 4 & $\begin{array}{l}\text { Station } \\
\quad 5\end{array}$ & 6 & 7 & 8 & 9 & $\begin{array}{c}\text { Yaquina Bay } \\
\text { reference }\end{array}$ \\
\hline 1980 & 15.4 & 16.0 & 16.0 & 18.4 & NT & 16.8 & $\mathrm{NT}$ & 19.4 & 19.0 & 19.6 \\
\hline 1983 & 18.2 & NT & 17.2 & 19.2 & 18.2 & 16.6 & 18.6 & 19.4 & 18.2 & 19.4 \\
\hline
\end{tabular}

collected from the Palos Verdes Shelf and Santa Monica Bay in 1980 and 1983 were not significantly different from the reference sediment.

\section{DISCUSSION}

Benthic conditions improved on the Palos Verdes Shelf between 1980 and 1983. The level of organic enrichment and the concentration of most measured contaminants decreased at all stations on the $60 \mathrm{~m}$ contow except the reference station in Santa Monica Bay. Four of 5 predictions concerning changes in the macrobenthos in response to the decline in sediment contamination were confirmed. Benthic abundance at Stn 6, the site of maximum stimulation in 1980, declined in 1983. With the exception of infaunal echinoderms, more sensitive species generally appeared closer to or became more abundant near the outfalls in 1983. The most pollution-tolerant species either disappeared or became less abundant near the outfalls in 1983. Evidence for acute sediment toxicity at stations within $3 \mathrm{~km}$ of the outfalls in 1980 was not found in 1983. The prediction that was not confirmed concerned an expected movement toward the outfalls of the area of maximum benthic stimulation. Instead, the site of maximum benthic species richness, density and biomass remained at Stn 6 , about $9 \mathrm{~km}$ from the outfalls in 1980 and 1983.

Characterization of species that became much less abundant at stations close to the outfalls as 'tolerant' is supported by Mearns \& Word's (1982) grouping of species for the Infaunal Index. Capitella spp. and Schistomeringos longicornis were dominants at Stns 1 , 2 , and 3 in 1980 and became much less abundant in 1983. Both species were placed by Mearns \& Word (1982) in Infaunal Index Group IV that includes species most abundant in areas heavily affected by sewage discharges.

Species that were found closer to or became more abundant near the outfalls in 1983 typically belong to Infaunal Groups II (Euphilomedes spp., Mediomastus californiensis, Axinopsida serricata) or III (Parvilucina tenuisculpta). Infaunal groups II and III are abundant in areas under slight or moderate influence of the discharges (Mearns \& Word 1982).

Some sensitive taxa, especially Amphiodia urtica and other infaunal echinoderms, were rare at all stations on the Shelf in both 1980 and 1983. Amphipods were more abundant near the outfalls in 1983, while their density declined significantly at sites further away on the Shelf and in Santa Monica Bay. Although the cause of this decline is uncertain, the similarity in amphipod density fluctuations at the reference station and at sites 9 to $15 \mathrm{~km}$ from the outfall suggests the changes occurred over a relatively large spatial scale.

Ecological changes close to the outfalls represent only a partial recovery of the benthos. Infaunal Group I dominates natural assemblages at $60 \mathrm{~m}$ in the southern California Bight (Mearns \& Word 1982). Species from this group were rarely collected near the outfalls. Although the structure of the benthic assemblage close to the outfalls improved in 1983, species richness, numbers of amphipods and echinoderms, numerical dominance, and Infaunal Index were significantly less there in 1983 than at the reference station. We therefore characterize conditions near the outfalls as changing from a major degradation in 1980 to a moderate degradation in 1983 (Table 3 ).

The significant reductions in benthic density and biomass at Stns 4, 6, and 8 also represent a partial recovery of the benthos relative to the reference station (Table 3). This decline in benthic stimulation was evident for most dominant species in the assemblage (Table 1). The only major exception was the increased abundance of Euphilomedes spp. Although the densities of Parvilucina tenuisculpta and Tharyx sp. A decreased by about $60 \%$ in 1983 , they remained the dominant species. The size of both individuals and populations of $P$. tenuisculpta is related to the degree of sediment organic enrichment near sewage outfalls (Fabrikant 1984).

The 1980 to 1983 reduction in sediment contamination on the Palos Verdes Shelf was correlated with macrobenthos alterations that are generally consistent with predictions based on the Pearson \& Rosenberg 
(1978) model. However, the expected spatial shift of the area of maximum benthic stimulation toward the outfalls did not occur in 1983. The concentration of most sediment contaminants at Stns 1, 3, and 4 in 1983 was greater than at the site of maximum benthic stimulation in 1980 (Stn 6, Table 4). Thus, the improvements in sediment quality may not have been sufficient to allow a major benthic stimulation at stations close to the outfalls in 1983.

We have used the terms stimulation and degradation to classify benthic conditions according to significant changes in species richness, number of individuals and biomass relative to a reference site (Table 3 ). This classification reflects the unimodal pattern of the biological gradient on the Palos Verdes Shelf (Fig. 2). In contrast, the chemical gradients on the Shelf typically show monotonic decreases with increasing distance from the outfalls (Table 4). The quantitative values of community parameters associated with different degrees of stimulation and degradation may be sitespecific, and perhaps time-specific, and should not be applied uncritically to other situations.

Although benthic stimulation results in more species, individuals, and biomass, these changes are not necessarily desirable from a management perspective. Differences in species composition between the stimulated assemblage on the Shelf and the reference assemblage may affect benthic coupling with pelagic segments of the ecosystem. The benthos of areas of the Christiaensen Basin in the New York Bight affected by sewage wastes is dense and productive, but little of the productivity is transferred to higher trophic levels (Boesch 1982). Also, the stimulated benthic assemblage is associated with a variety of sediment contaminants that have the potential for adverse ecological effects (Table 4).

The elimination of acute sediment toxicity, as determined by the phoxocephalid amphipod bioassay, at Stns 1 and 3 between 1980 and 1983 provides additional evidence of the improvement of ecological conditions on the Shelf. However, the absence of acute sediment toxicity does not demonstrate the absence of benthic degradation (Swartz et al. 1985b). Although some amphipods were found at Stn 1 for the first time in 1983, phoxocephalid amphipods were no closer to the outfalls than Stn 4 (Table 2).

Substantial reductions in the mass emission of organic materials and chemical contaminants from the LACSD outfalls are consistent with the improvement in sediment quality on the Palos Verdes Shelf. The benthic accumulation of chemicals released from sewage outfalls is affected by many physical and biogeochemical processes. Exactly proportional changes in effluent and sediment concentrations should not be expected. However, with the exceptions
Table 6. Comparison of changes between 1980 and 1983 in the mass emission of materials from the Los Angeles County sewage outfalls and sediment concentrations at Stn 1 on the Palos Verdes Shelf, California. Mass emission data from Schafer $(1982,1984)$

\begin{tabular}{|c|c|c|}
\hline \multirow[t]{2}{*}{ Parameter } & \multicolumn{2}{|c|}{ Percent change } \\
\hline & Mass emission & Stn 1 sediment \\
\hline BOD & -20.0 & -71.1 \\
\hline Oil \& grease & -18.0 & -66.7 \\
\hline Ammonia & -3.0 & -16.7 \\
\hline Cadmium & -28.0 & -18.8 \\
\hline Chromium & -56.1 & -19.1 \\
\hline Copper & -36.8 & -52.5 \\
\hline Nickel & -38.0 & -20.0 \\
\hline Lead & -35.5 & -42.9 \\
\hline Zinc & -18.1 & +2.3 \\
\hline DDT & -66.2 & $+19.7^{\circ}$ \\
\hline \multicolumn{3}{|c|}{$\begin{array}{l}\text { - Change calculated for DDE, the major DDT } \\
\text { metabolite at Stn } 1\end{array}$} \\
\hline
\end{tabular}

of DDT and zinc, there were corresponding reductions in the LACSD mass emission and Palos Verdes sediment concentration of most parameters for which comparative data are available (Table 6). Most of the DDT and its metabolites on the Shelf were introduced prior to source control in 1970 when the annual mass emission was about 100 metric tons (LACSD 1981). By 1980 the annual emission rate was reduced to $542 \mathrm{~kg}$ (Schafer 1980). The further $66.2 \%$ reduction to $183 \mathrm{~kg}$ DDT $\mathrm{yr}^{-1}$ in 1983 apparently had little influence on the sediment concentration of previously discharged DDT and its metabolites.

Two other factors that have been cited as possible causes of improvements in sediment contamination near large southern California sewage outfalls are sediment bioturbation and sediment scouring by bottom currents or wave action (Greene 1976a, b, Stull et al. 1986). Burrowing, feeding and respiratory activities of the echiuran worm Listriolobus pelodes may have caused a reduction in the areal extent of anaerobic sediment on the Palos Verdes Shelf from 1974 to 1977 (Stull et al. 1986). L. pelodes dominated the benthic biomass on the Shelf during that period. This worm was present at our stations in 1980 and 1983, but ranked among the 5 most abundant species only at Stn 3 in 1980 (Table 1).

Unusually severe storms affected the southern California coastal zone several times during the winter of 1982-83 (Dayton \& Tegner 1984, Gunnill 1985). These storms occurred during an El Nin̄o warm-water period whose impact on the productivity of the southern California Bight could also have affected the benthos of the Palos Verdes Shelf. The storm waves had heights exceeding $3 \mathrm{~m}$ and periods exceeding $20 \mathrm{~s}$ 
(Seymour \& Sonv cited by Dayton \& Tegner 1984). Such waves are capable of resuspending sediments at $60 \mathrm{~m}$ and could account for a reduction in contaminant concentrations in the surficial sediment layer (W. P. Muellenhoff pers. comm.). The occurrence of terrestrial plant debris at the reference Stn 9 may have resulted from stormwater runoff into Santa Monica Bay. Although this debris may have affected sediment chemistry, it had no obvious impact on the macrobenthos or sediment toxicity.

Reductions in BOD and chemical mass emissions from the LACSD outfalls, El Niño, and the 1982-83 winter storms may have all contributed to benthic improvements on the Palos Verdes Shelf. If the decline in mass emissions was the dominant factor, these improvements should persist in the future. If the $\mathrm{El}$ Niño or storms were responsible, the partial recovery of the Shelf benthos may be a temporary phenomenon.

Acknowledgements. We thank Don Baumgartner, Henry Lee, Jerry Pesch, Steve Ferraro, and Bob Whitlatch for their reviews of the manuscript; George Ditsworth, Kathy Sercu, Janet Lamberson, and Jill Jones for technical support; and the crew of the R/V Marine Surveyor for assistance during the field collections.

\section{LITERATURE CITED}

Anger, K. (1975). On the influence of sewage pollution on inshore benthic communities in the south of Kiel Bay. Helgoländer Meeresunters. 27: 408-438

Bascom, W. (1978). Life in the bottom. Annual Report 1978 , Southern California Coastal Water Research Project, El Segundo, California, 1978, p. 57-80

Bascom, W. (1982). The effects of waste disposal on the coastal waters of Southern California. Environ. Sci. Technol. 16: 226A-236A

Bascom, W., Mearns, A. J., Word, J. K. (1978). Establishing boundaries between normal, changed, and degraded areas. Annual Report 1978, Southern California Coastal Water Research Project, El Segundo, California, p. 81-94

Boesch, D. F. (1977). Application of numerical classification in ecological investigations of water pollution. Ecological Research Series 600/3-77-033, U.S. Environmental Protection Agency, Washington, D.C., p. 1-115

Boesch, D. F. (1982). Ecosystem consequences of alterations of benthic community structure and function in the New York Bight. In: Mayer, G. F. (ed.) Ecological stress and the New York Bight: science and management. Estuarine research federation, Columbia, South Carolina, p. $543-568$

Dayton, P. K., Tegner, M. J. (1984). Catastrophic storms, El Niño, and patch stability in a southern California kelp community. Science 224: 283-285

Fabrikant, R. (1984). The effect of sewage effluent on the population density and size of the clam Parvilucina tenuisculpta. Mar Pollut. Bull. 15: 249-253

Grassle, J. P., Grassle, J. F. (1976). Sibling species in the marine pollution indicator Capitella (Polychaeta). Science 192: $567-569$

Greene, C. S. (1976a). Response and recovery of the benthos at Orange County. Annual Report 1976, Southern Califor- nia Coastal Water Research Project, El Segundo, California, p. 197-203

Greene, C. S. (1976b). Partial recovery of the benthos at Palos Verdes. Annual Report 1976, Southern California Coastal Water Research Project, El Segundo, California, p 205-210

Gunnill, F. C. (1985). Population fluctuations of seven macroalgae in southern California during 1981-1983 including effects of severe storms and an El Niño. J. exp. mar Biol. Ecol. 85: 149-164

Hershelman, G. P., Schafer, H. A., Jan, T.-K., Young, D. R. (1981). Metals in marine sediments near a large California municipal outfall. Mar. Pollut. Bull. 12: 131-134

Lance, G. N., Williams, W. T. (1966). A generalized sorting strategy for computer classifications. Nature, Lond. 212: 218

Los Angeles County Sanitation Districts (LACSD). (1981). Ocean monitoring and research. Annual Report 1980-1981, Los Angeles County Sanitation Districts, Whittier, California, p. 19-150

Mearns, A. J., Word, J. Q. (1982). Forecasting effects of sewage solids on marine benthic communities. In: Mayer, G. F. (ed.) Ecological stress and the New York Bight: science and management. Estuarine Research Federation, Columbia, South Carolina, p. 495-512

Pearson, T. H., Rosenberg, R. (1978). Macrobenthic succession in relation to organic enrichment and pollution of the marine environment. Oceanogr. mar. Biol. A. Rev. 16: $229-311$

Southern California Coastal Water Research Project. (1973). The ecology of the Southern California Bight: Implications for water quality management. SCCWRP TR104, Southern Califormia Coastal Water Research Project, El Segundo, California, p. 1-531

Schafer, H. A. (1978). Characteristics of municipal wastewater discharges, 1977. Annual Report 1978, Southern California Coastal Water Research Project, El Segundo, California, p. 97-101

Schafer, H. A. (1980). Characteristics of municipal wastewater Biennial Report 1979-1980, Southern California Coastal Water Research Project, Long Beach, California, p. $235-239$

Schafer, H. A. (1984). Characteristics of municipal wastewaters, 1982-83. Biennial Report 1983-84, Southern California Coastal Water Research Project, Long Beach, California, p. 11-19

Smith, G. B. (1974). Some effects of sewage discharge to the marine environment. Ph. D. dissertation, Univ. of California, San Diego

Smith, R. W., Greene, C. S. (1976). Biological communities near a submarine outfall. J. Wat. Pollut. Control Fed. 48: $1894-1912$

Sokal, R. R., Rohlf, F. J. (1981). Biometry, 2nd edn. W. H. Freeman, San Francisco

Steel, R. G. D., Torrie, J. H. (1960). Principals and procedures of statistics. McGraw-Hill, New York

Stull, J. K., Haydock, C. I., Montagne, D. E. (1986). Effects of Listriolobus pelodes (Echiura) on coastal shelf benthic communities and sediments modified by a major California wastewater discharge. Estuar. coast. Shelf Sci. 22: $1-17$

Swartz, R. C., Schults, D. W., Ditsworth, G. R., DeBen, W. A., Cole, F. A. (1985a). Sediment toxicity, contamination, and macrobenthic communities near a large sewage outfall. In: Boyle, T. P. (ed.) Validation and predictability of laboratory methods for assessing the fate and effects of contaminants in aquatic ecosystems. ASTM STP 865. 
American Society for Testing and Materials, Philadelphia, p. $152-175$

Swartz, R. C., DeBen, W. A., Jones, J. K. P., Lamberson, J. O., Cole, F. A. (1985b). Phoxocephalid amphipod bioassay for marine sediment toxicity. In: Cardwell, R. D., Purdy, R., Bahner, R. C. (ed.) Aquatic toxicology and hazard assessment. Seventh Symposium, ASTM STP 854, American Society for Testing and Materials, Philadelphia, p. $284-307$

Word, J. Q. (1978). The infaunal trophic index. Annual Report
1978, Southern California Coastal Water Research Project, El Segundo, California, p. 19-39

Word, J. Q. (1980). Classification of benthic invertebrates into infaunal trophic index feeding groups. Biennial Report 1979-1980, Southern California Coastal Water Research Project, Long Beach, California, p. 103-121

Word, J. Q., Mearns, A. J. (1978). The 60-meter control survey. Annual Report 1978, Southern Califomia Coastal Water Research Project, El Segundo, California, p. 41-56

This article was presented by Professor J. Gray; it was accepted for printing on April 2, 1986 